\title{
The boostless bootstrap and BCFW momentum shifts
}

\author{
David Stefanyszyn and Jakub Supeł \\ Department of Applied Mathematics and Theoretical Physics, \\ Centre for Mathematical Sciences, University of Cambridge, \\ Wilberforce Road, Cambridge CB3 OWA, U.K. \\ E-mail: d.stefanyszyn@damtp.cam.ac.uk, js2154@cam.ac.uk
}

ABSTRACT: In a recent paper [1], three-particle interactions without invariance under Lorentz boosts were constrained by demanding that they yield tree-level four-particle scattering amplitudes with singularities as dictated by unitarity and locality. In this brief note, we show how to obtain an independent verification and consistency check of these boostless bootstrap results using BCFW momentum shifts. We point out that the constructibility criterion, related to the behaviour of the deformed amplitude at infinite BCFW parameter $z$, is not strictly necessary to obtain non-trivial constraints for the three-particle interactions.

Keywords: Scattering Amplitudes, Space-Time Symmetries, Gauge Symmetry

ARXiv EPrint: 2009.14289 


\section{Contents}

1 Introduction 1

2 Spinor helicity formalism and BCFW deformations 3

3 Constructibility criterion $\quad 5$

4 Constraining three-particle interactions $\quad 6$

$\begin{array}{llr}5 & \text { Summary } & 8\end{array}$

\section{Introduction}

The on-shell approach to computing scattering amplitudes had led to tremendous advances in our understanding of gauge theory and gravity [2-4]. Weinberg's seminal papers from the 60 's $[5,6]$ showed that the combination of Poincare invariance and unitarity uniquely picks out Maxwell's equations as the description of a massless spin-1 particle (photon) and Einstein's equations as the description of a massless spin-2 particle (graviton), at tree-level. In addition, charge conservation and the equivalence principle follow from consistency of scattering amplitudes involving photons and gravitons, respectively.

Since then, there have been many attempts to constrain or bootstrap interactions using a purely on-shell approach, by invoking the principles of unitarity, locality and causality. One of these attempts is the technique known as BCFW deformations [7], in which some of the external momenta are deformed by a complex parameter $z$. Consideration of the analytic structure of the amplitude as a function of $z$ often imposes non-trivial constraints on interactions [8], as we will explain in detail in section 2. Constraints can also be derived in another way [9-11], without deforming the amplitude, but rather by demanding consistent factorisation. Here one simply writes down the most general form of the tree-level four-particle amplitude, and demands that poles only arise due to intermediate (exchanged) particles going on-shell, with the corresponding residues given by a product of on-shell three-particle amplitudes.

Notable results of this bootstrap programme include the observations that long-range forces cannot be mediated by particles with spin $\geq 3$, Yang-Mills (YM) is the unique theory of multiple massless spin-1 particles, General Relativity (GR) is the unique lowenergy theory of a massless spin-2 particle, and the existence of massless spin- $3 / 2$ particles requires gravity and supersymmetry leading uniquely to supergravity ${ }^{1}[8,10]$. This on-shell

\footnotetext{
${ }^{1}$ One can also show that there is an upper limit on the number of spin-3/2 particles that a unitary theory can contain [10].
} 
approach shows that the low-energy properties of Poincare invariant theories are virtually inevitable.

Given such spectacular success, attention has recently turned to applying similar methods to spatial correlation functions which are the fundamental observables in cosmology, see [12-28] and references therein. These cosmological correlators live on the late-time boundary of an approximate de Sitter spacetime and encode details about the bulk spacetime through their dependence on spatial momenta. A key ingredient in this cosmological bootstrap is the fact that correlators contain flat-space scattering amplitudes in the residues of their total-energy poles [29, 30] (for a detailed proof see [28]). These amplitudes form part of the theoretical data required to bootstrap the corresponding correlators, and therefore the cosmological bootstrap programme requires us to have a solid understanding of flat-space scattering amplitudes.

Much effort has so far focused on correlators fixed by de Sitter symmetries (or conformal symmetries on the boundary). Intuitively, taking the cosmological bootstrap programme beyond exact de Sitter symmetries in order to construct inflationary correlators, requires taking the $S$-matrix bootstrap beyond exact Poincaré symmetries. Motivated by this, a recent paper [1] derived the singular parts of tree-level four-particle amplitudes where the free propagators are assumed to be massless and Poincaré invariant, i.e. all dispersion relations are linear with each particle propagating at the same speed, but with interactions that are allowed to break Lorentz boosts. Within this set-up, analytically continued threeparticle amplitudes are fixed by the helicities of the external particles up to an almost arbitrary function of their energies and these functions are then constrained by demanding consistent factorisation, but without making use of BCFW shifts. The most interesting results from this boostless bootstrap are [1]:

- When there is a graviton in the spectrum, all three-particle interactions must reduce to their Poincaré invariant form, even those that do not involve the graviton itself. ${ }^{2}$ Universal coupling of gravity to all other particles is then recovered.

- Low-energy self-interactions of a photon must vanish. The leading allowed operator has six derivatives and is therefore mass dimension-9.

- There exists at least one large class of boost-breaking theories involving scalars, spin-1/2 fermions and photons (boost-breaking massless QED). The corresponding Lagrangians contain generalised, boost-breaking gauge redundancies.

In the Poincaré invariant cases, all the results derived by imposing consistent factorisation have also been reached via the BCFW formalism. Likewise, in this paper we show that the results of [1] can be derived using BCFW shifts as a tool to automate consistent factorisation and therefore provide a neat consistency check. In section 2 we briefly introduce the spinor helicity formalism that we will use throughout, present general three-particle amplitudes for boost-breaking theories, and briefly review BCFW momentum shifts. In section 3

\footnotetext{
${ }^{2}$ Related results based on field-theoretic methods have been derived in [31-33]. See also [34] where Lorentz boosts arise in the presence of soft gravitons.
} 
we argue that BCFW shifts remain a useful tool for non-constructible theories by making a distinction between accessible and inaccessible singularities. In this case, while the fourparticle amplitudes are not completely fixed by the three-particle ones, we can still use BCFW shifts to constrain the latter. We illustrate this in section 4 for boost-breaking theories of self-interacting spin- $S$ particles. We end the paper with some concluding remarks.

\section{Spinor helicity formalism and BCFW deformations}

We work in four spacetime dimensions and use the spinor helicity formalism to present amplitudes in a compact form. In this formalism, a complex null four-momentum $p^{\mu}$ is represented as a product of two-component spinors as ${ }^{3}$

$$
p_{\alpha \dot{\alpha}}=\sigma_{\alpha \dot{\alpha}}^{\mu} p_{\mu}=\lambda_{\alpha} \tilde{\lambda}_{\dot{\alpha}}
$$

where $\sigma_{\alpha \dot{\alpha}}^{\mu}$ are the Pauli matrices and the undotted and dotted indices transform in the spinor representations of the Lorentz group i.e. $(1 / 2,0)$ and $(0,1 / 2)$ respectively. Throughout we follow the conventions of [35] and we assume that each particle satisfies $p^{\mu} p_{\mu}=E^{2}-\mathbf{p}^{2}=0$ on-shell. When boosts are broken, amplitudes are constructed from $\mathrm{SO}(3)$ invariant quantities rather than $\mathrm{SO}(1,3)$ invariant ones. In [1], it was shown that such three-particle amplitudes are functions of the following objects:

- "angle" brackets: $\langle i j\rangle=\epsilon^{\alpha \beta} \lambda_{\alpha}^{(i)} \lambda_{\beta}^{(j)}$,

- "square" brackets: $[i j]=\epsilon^{\dot{\alpha} \dot{\beta}} \tilde{\lambda}_{\dot{\alpha}}^{(i)} \tilde{\lambda}_{\dot{\beta}}^{(j)}$,

- energies: $E_{i}$.

Here latin indices label the external particles: $i, j=1,2,3$. We remind the reader that the spinors are not grassmanian and therefore the angle and square brackets are antisymmetric. By demanding that the amplitudes scale in the appropriate way under helicity transformations, on-shell, non-perturbative three-particle amplitudes take the form [1]

$$
\mathcal{A}_{3}= \begin{cases}\langle 12\rangle^{d_{3}}\langle 23\rangle^{d_{1}}\langle 31\rangle^{d_{2}} F^{H}\left(E_{1}, E_{2}, E_{3}\right), & h<0, \\ {[12]^{-d_{3}}[23]^{-d_{1}}[31]^{-d_{2}} F^{A H}\left(E_{1}, E_{2}, E_{3}\right),} & h>0,\end{cases}
$$

where $d_{i}=2 h_{i}-h, h_{i}$ is the helicity of the $i^{\text {th }}$ particle and $h$ is the sum of the helicities (if $h=0$, the amplitude can be a sum of the two expressions.) The presence of functions ${ }^{4}$ $F^{H}, F^{A H}$ (which depend on the helicities) reflects the fact that Lorentz boosts are no longer an assumed symmetry. The Poincaré invariant amplitudes are recovered when these functions are constant. As energy is conserved, we will often consider the $F$ 's as functions of two arguments only. As an example, the three-particle amplitude for three incoming gravitons, two with negative helicity and one with positive helicity, is

$$
\mathcal{A}_{3}\left(1^{-2}, 2^{-2}, 3^{+2}\right)=\left(\frac{\langle 12\rangle^{3}}{\langle 23\rangle\langle 31\rangle}\right)^{2} F^{H}\left(E_{1}, E_{2}\right) .
$$

In this case, Bose symmetry dictates that $F^{H}\left(E_{1}, E_{2}\right)=F^{H}\left(E_{2}, E_{1}\right)$.

\footnotetext{
${ }^{3}$ The inverse of this equation is $p^{\mu}=\frac{1}{2}\left(\bar{\sigma}^{\mu}\right)^{\dot{\alpha} \alpha} p_{\alpha \dot{\alpha}}$ and so the energy of a particle in terms of the spinors is $E=\frac{1}{2}\left(\bar{\sigma}^{0}\right)^{\dot{\alpha} \alpha} \lambda_{\alpha} \tilde{\lambda}_{\dot{\alpha}}$.

${ }^{4}$ The superscripts refer to holomorphic and anti-holomorphic kinematic configurations [8].
} 
Now, to be consistent with unitarity and locality, a tree-level four-particle amplitude must factorise into a product of on-shell three-particle amplitudes on each of its poles. Poles correspond to exchanged particles going on-shell and consistent factorisation dictates that, for example,

$$
\lim _{s \rightarrow 0}\left(s \mathcal{A}_{4}\right)=\mathcal{A}_{3}(1,2,-I) \times \mathcal{A}_{3}(3,4, I),
$$

where $s=\left(p_{1}+p_{2}\right)^{2}$ is the propagator of the exchanged particle which is labelled by $I$. Analogous relations hold when $t=\left(p_{1}+p_{3}\right)^{2} \rightarrow 0$ and $u=\left(p_{1}+p_{4}\right)^{2} \rightarrow 0$. For future reference, in terms of the spinors these Mandelstam variables are given by $s=\langle 12\rangle[12]=\langle 34\rangle[34]$, $t=\langle 13\rangle[13]=\langle 24\rangle[24]$ and $u=\langle 14\rangle[14]=\langle 23\rangle[23]$. Requiring the amplitude to factorise correctly on each pole is often highly non-trivial $[1,8-11]$ since, for example, the $s$-channel residue can contain poles in $t$ and $u$ which then need to be interpreted as propagation of a particle in those channels. This is beautifully illustrated for multiple spin-1 particles where consistency in each channel requires the coupling constants to satisfy the Jacobi identity.

In [8], Benincasa and Cachazo elegantly used BCFW shifts [7] to formally assess this consistency for a number of tree-level four-particle amplitudes. The simplest BCFW shift takes two particles $i$ and $j$ and deforms their energies and momenta according to

$$
\lambda^{(i)}(z)=\lambda^{(i)}+z \lambda^{(j)}, \quad \tilde{\lambda}^{(j)}(z)=\tilde{\lambda}^{(j)}-z \tilde{\lambda}^{(i)},
$$

with all other spinors kept fixed. This choice preserves the on-shell conditions for both particles as well as energy-momentum conservation. The deformed amplitude $\mathcal{A}_{4}^{(i, j)}(z)$ is a rational function of the complex parameter $z$ and thus can be fully deduced from knowledge of its poles, residues and behaviour at infinity. It takes the form

$$
\mathcal{A}_{4}^{(i, j)}(z)=\sum_{n} \frac{\operatorname{res}_{z=z_{n}} \mathcal{A}_{4}^{(i, j)}(z)}{z-z_{n}}+B^{(i, j)}(z)
$$

where the boundary term $B^{(i, j)}(z)$ is regular in the entire complex plane. For four-particle amplitudes, only two poles can be reached by a given deformation (since $p_{i}+p_{j}$ is independent of $z$ ) and as we remarked above, the corresponding residues are evaluated from the three-particle amplitudes alone. For example, summing over the possible helicities of the exchanged particle, for $\mathcal{A}_{4}^{(1,2)}(z)$ the two poles that can be reached are $z_{t}$ and $z_{u}$ and we have

$$
\mathcal{A}_{4}^{(1,2)}(z)=\sum_{h_{I}} \frac{A_{3}(\hat{1}, 3,-\hat{I}) A_{3}(\hat{2}, 4, \hat{I})}{t(z)}+\sum_{h_{I}} \frac{A_{3}(\hat{1}, 4,-\hat{I}) A_{3}(\hat{2}, 3, \hat{I})}{u(z)}+B^{(1,2)}(z),
$$

where a hat indicates that the particle has had one of its spinors deformed and evaluated at the appropriate pole. We have $t(z)=\left(p_{1}(z)+p_{3}\right)^{2}=\langle 13\rangle[13]+z\langle 23\rangle[13]$ and $u(z)=\left(p_{1}(z)+p_{4}\right)^{2}=\langle 14\rangle[14]+z\langle 24\rangle[14]$ and therefore the locations of the poles are

$$
z_{t}=-\frac{\langle 13\rangle}{\langle 23\rangle}, \quad z_{u}=-\frac{\langle 14\rangle}{\langle 24\rangle} .
$$

$\mathcal{A}_{4}^{(i, j)}(0)$ corresponds to the amplitude for unshifted momenta, and then constraints on three-particle couplings can be derived by demanding that distinct $\mathcal{A}_{4}^{(i, j)}(0)$ coincide at $z=0[8]$ i.e.

$$
\mathcal{A}_{4}^{(i, j)}(0)=\mathcal{A}_{4}^{(k, l)}(0) \quad \forall \quad i, j, k, l .
$$

This is the four-particle test. 


\section{Constructibility criterion}

In its original formulation, the above described method is reserved for constructible theories for which $B^{(i, j)}(z)$ vanishes. In this case the singular parts of undeformed amplitudes can be compared with one another and the full four-particle amplitude is determined by the three-particle ones. Since $B^{(i, j)}(z)$ is regular, it is sufficient to prove that the amplitude tends to zero as $z \rightarrow \infty$. This is usually a non-trivial matter, necessitating a reference to the Lagrangian and a detailed counting of powers of momenta. Fortunately, many theories describing nature are constructible including, most notably, YM $[7,36]$ and GR [37] (scalar field theories are not constructible in the sense described above. This has lead to new, interesting momentum shifts and on-shell recursion relations being derived for scalar theories with non-linearly realised symmetries $[38,39]$ ).

However, for the boost-breaking amplitudes of interest here, it is unlikely that $B^{(i, j)}(z)$ would vanish, since the unknown functions of energies will in general contribute positive powers of $z$ to the tree-level amplitude. Indeed, for both particles $i$ and $j$, the deformation of their energies is linear in $z$ and so the divergence at large $z$ gets worse as additional powers of energy are included:

$$
\begin{aligned}
& E_{i}(z)=E_{i}(0)+\frac{z}{2}\left(\bar{\sigma}^{0}\right)^{\dot{\alpha} \alpha} \lambda_{\alpha}^{(j)} \tilde{\lambda}_{\dot{\alpha}}^{(i)}, \\
& E_{j}(z)=E_{j}(0)-\frac{z}{2}\left(\bar{\sigma}^{0}\right)^{\dot{\alpha} \alpha} \lambda_{\alpha}^{(j)} \tilde{\lambda}_{\dot{\alpha}}^{(i)} .
\end{aligned}
$$

The BCFW method can still be useful for non-constructible theories, however. One possibility is to introduce a distinction between accessible and inaccessible singularities of $\mathcal{A}_{4}^{(i, j)}(z)$, along the lines of [40]. We say a singularity is accessible via a deformation of momenta $i$ and $j$ if this singularity is approached as $z \rightarrow z_{*}$ for some $z_{*}$. Otherwise we say it is inaccessible. The regular term $B^{(i, j)}(z)$, by definition, cannot have any singularities in the $z$-plane and therefore cannot contribute to any residues of the accessible singularities of $\mathcal{A}_{4}^{(i, j)}(z)$. But it may exhibit inaccessible singularities. As an illustration of this distinction, consider a single scalar theory which is famously non-constructible. In the absence of additional global charges, the three-particle amplitude is a non-zero constant, $\mathcal{A}_{3}=g$, and so we have

$$
\begin{aligned}
& \mathcal{A}_{4}^{(1,2)}(0)=g^{2}\left(\frac{1}{t}+\frac{1}{u}\right)+B^{(1,2)}(0), \\
& \mathcal{A}_{4}^{(1,4)}(0)=g^{2}\left(\frac{1}{s}+\frac{1}{t}\right)+B^{(1,4)}(0) .
\end{aligned}
$$

The consistency condition $\mathcal{A}_{4}^{(1,2)}(0)=\mathcal{A}_{4}^{(1,4)}(0)$ can be satisfied by choosing $B^{(1,2)}(z)=\frac{g^{2}}{s}$ and $B^{(1,4)}(z)=\frac{g^{2}}{u}$, since these two functions do not have any accessible singularities with regards to their own deformations.

In the following section we will constrain boost-breaking amplitudes using the fact that the regular term $B^{(i, j)}(z)$ does not have any accessible singularities. We will see that for spinning particles, we can derive the highly non-trivial constraints first found in [1]. 


\section{Constraining three-particle interactions}

In this section we will constrain three-particle interactions for theories of a single spin$S$ particle with integer $S$. We will derive the constraints first presented in [1]. We also checked that the BCFW techniques allow us to recover other results in [1], namely those of (gravitational) Compton scattering and the full analysis for a scalar or a photon coupled to gravity. Those calculations contain only minor differences compared with what is presented below so we omit the details in favour of brevity. We remind the reader that we do not impose boost invariance, but only demand that the free theory is Poincaré invariant, with the on-shell condition $E^{2}-\mathbf{p}^{2}=0$ for each particle.

Consider the amplitude $\mathcal{A}_{4}\left(1^{+S} 2^{-S} 3^{+S} 4^{-S}\right)$, where superscripts denote the helicities of incoming particles of some integer spin $S$. We will impose matching conditions between deformations $(1,2)$ and $(1,4)$. First consider $(1,2)$. Using the expressions for ${ }^{5}$ $A_{3}\left(1^{+S}, 2^{+S}, 3^{-S}\right)$ and $A_{3}\left(1^{-S}, 2^{-S}, 3^{+S}\right)$ given in $(2.2)$, and $^{6}$

$$
\begin{aligned}
p_{1}\left(z_{t}\right)+p_{3} & =\frac{[13]}{[14]} \lambda^{(3)} \tilde{\lambda}^{(4)}, \\
p_{1}\left(z_{u}\right)+p_{4} & =\frac{[14]}{[13]} \lambda^{(4)} \tilde{\lambda}^{(3)},
\end{aligned}
$$

to eliminate all copies of $\lambda^{(I)}$ and $\tilde{\lambda}^{(I)}$, which are the spinors associated with the exchanged particle ${ }^{7}$ we find

$$
\mathcal{A}_{4}^{(1,2)}(0)=B^{(1,2)}(0)+\left(\frac{1}{t} F_{\hat{1}, 3} F_{\hat{2}, 4}+\frac{1}{u} F_{\hat{1},-\hat{1}-4} F_{\hat{2},-\hat{2}-3}\right)\left(\frac{[13]^{2}\langle 24\rangle^{2}}{s}\right)^{S} .
$$

In the $u$-channel we have summed over the two possibilities for the helicity configuration of the exchanged particle but given (4.2), only one of these is non-zero. To keep formulae compact, here we have introduced subscripts to the $F$ 's to denote their arguments e.g. $F\left(E_{i}, E_{j}\right) \equiv F_{i, j}$ and $F\left(E_{i}, E_{j}+E_{k}\right) \equiv F_{i, j+k}$. Again, hats denote deformed objects evaluated at the appropriate points e.g. in the $1 / t$ coefficient, $F_{\hat{1}, 3} \equiv F\left(\hat{E}_{1}\left(z_{t}\right), E_{3}\right)$ where $\hat{E}_{1}\left(z_{t}\right)$ is the deformed energy of particle 1 evaluated at $z=z_{t}$. Likewise, in the $1 / u$ coefficient, hatted energies are evaluated at $z=z_{u}$. We have also removed the $H / A H$ superscripts since the functions are identical, due to parity, up to an inconsequential overall sign [1].

Now, we can also write

$$
\mathcal{A}_{4}^{(1,2)}(0)=\tilde{B}^{(1,2)}(0)+\left(\frac{1}{t} F_{1,3} F_{2,4}+\frac{1}{u} F_{1,-1-4} F_{2,-2-3}\right)\left(\frac{[13]^{2}\langle 24\rangle^{2}}{s}\right)^{S},
$$

\footnotetext{
${ }^{5}$ These amplitudes arise from the leading order couplings. Higher-dimension operators give rise to the $A_{3}\left(1^{+S}, 2^{+S}, 3^{+S}\right), A_{3}\left(1^{-S}, 2^{-S}, 3^{-S}\right)$ amplitudes but we don't consider these here. We refer the reader to [1] for a discussion on these amplitudes.

${ }^{6}$ Here we have assumed [13] and [14] are non-zero, and therefore $t=0$ and $u=0$ are approached as $\langle 13\rangle=0$ and $\langle 14\rangle=0$ respectively (or as $[24]=0$ and $[23]=0$ respectively, by momentum conservation).

${ }^{7}$ For example, in the $t$-channel we set $\lambda^{(I)}=\alpha \lambda^{(3)}$ and $\tilde{\lambda}^{(I)}=\beta \tilde{\lambda}^{4}$ with $\alpha \beta=\frac{[13]}{[14]}$. When computing the residue, $\alpha$ and $\beta$ only appear in the product $\alpha \beta$.
} 
where here we dropped the hat above all the energies, which indicates that the expression is evaluated at their undeformed values. This can be justified as follows. We assume that the F's can be Taylor expanded around the undeformed energies. The deformed energies are

$$
\begin{aligned}
& \hat{E}_{1}\left(z_{t}\right)=E_{1}-\frac{t}{2\langle 23\rangle[13]}\left(\bar{\sigma}^{0}\right)^{\dot{\alpha} \alpha} \lambda_{\alpha}^{(2)} \tilde{\lambda}_{\dot{\alpha}}^{(1)}, \\
& \hat{E}_{2}\left(z_{t}\right)=E_{2}+\frac{t}{2\langle 23\rangle[13]}\left(\bar{\sigma}^{0}\right)^{\dot{\alpha} \alpha} \lambda_{\alpha}^{(2)} \tilde{\lambda}_{\dot{\alpha}}^{(1)},
\end{aligned}
$$

with similar expressions evaluated at $z=z_{u}$. Here we see that potential new singularities generated by the deformed energies are all inaccessible, as they correspond to the vanishing of $\langle 23\rangle$ or [13], but these do not depend on $z$. Moreover, only the leading term in the Taylor expansion will exhibit accessible singularities, since in all subleading terms $t$ and $u$ will be cancelled out. We can therefore simply absorb all subleading terms into $B^{(1,2)}$, thus introducing $\tilde{B}^{(1,2)}$ that still does not contain any terms singular in $t$ or $u$. Although it could become singular in some kinematic configurations, especially at $s=0$, that is not a problem, because this singularity is inaccessible and we only demand that $\tilde{B}^{(1,2)}$, for those configurations for which it can be defined, does not have any singularities as a function of $z$.

We now play the same game for the $(1,4)$ deformation which amounts to interchanging particles 2 and 4 . We have

$$
\mathcal{A}_{4}^{(1,4)}(0)=\tilde{B}^{(1,4)}(0)+\left(\frac{1}{t} F_{1,3} F_{4,2}+\frac{1}{s} F_{1,-1-2} F_{4,-4-3}\right)\left(\frac{[13]^{2}\langle 24\rangle^{2}}{u}\right)^{S} .
$$

We discussed the $S=0$ case earlier where we showed that equating $\mathcal{A}_{4}^{(1,2)}(0)$ and $\mathcal{A}_{4}^{(1,4)}(0)$ requires us to make certain choices for the boundary terms. Let us now consider $S>0$ with $S$ an integer. We see that $\mathcal{A}_{4}^{(1,2)}(0)$ in (4.4) contains terms proportional to $1 /\left(t s^{S}\right)$ and $1 /\left(u s^{S}\right)$ which are both singular in more than one Mandelstam variable and thus cannot be accounted for or modified by $\tilde{B}^{(1,2)}(0)$ nor $\tilde{B}^{(1,4)}(0)$. A similar observation applies to $\mathcal{A}_{4}^{(1,4)}(0)$ in (4.7). Thus, by matching the amplitudes we find the necessary condition

$$
\frac{a}{s^{S} t}+\frac{b}{s^{S} u}=\frac{c}{u^{S} t}+\frac{d}{u^{S} s},
$$

where

$$
\begin{aligned}
a & =F_{1,3} F_{2,4}, \\
b & =F_{1,-1-4} F_{2,-2-3}, \\
c & =F_{1,3} F_{4,2}, \\
d & =F_{1,-1-2} F_{4,-4-3} .
\end{aligned}
$$

Recalling that $s+t+u=0$, this constraint, given that it must be valid for all kinematics, is equivalent to

$$
a u^{S}-b(s+u) u^{S-1}-c s^{S}+d(s+u) s^{S-1}=0 .
$$

For $S=1$ we therefore have $a=(b-d)=-c$, or equivalently,

$$
F_{1,3} F_{2,4}-F_{1,-1-4} F_{2,-2-3}+F_{1,-1-2} F_{4,-4-3}=0,
$$


which is simply an alternative form of (4.25) from [1]. Assuming that the F's are polynomials, in [1] it was shown that the only solution to this system is $F \equiv 0$ once we impose that the $S=1$ functions are alternating polynomials as dictated by Bose symmetry. ${ }^{8}$ We therefore see that the leading order three-particle interactions for three-photons must vanish, as is the case for Poincaré invariant theories. For $S=2$ we require $a=b=c=d$, or equivalently,

$$
F_{1,3} F_{2,4}=F_{1,-1-4} F_{2,-2-3}=F_{1,-1-2} F_{4,-4-3}
$$

which gives rise to the constraints $(4.31)-(4.33)$ from [1] once we use the fact that the $S=2$ functions are symmetric in their arguments by Bose symmetry ${ }^{9}$ (this also makes the $a=c$ constraint trival). In [1] it was shown that the only solution to this system is $F=$ constant and so again the three-particle interactions are reduced to their Poincaré invariant form, but this time the amplitudes are non-zero and are those of GR. Finally, for $S>2$, it is simple to see that $a=b=c=d=0$ is required and therefore there are no consistent three-particle interactions for these massless, higher-spin particles even when boosts are broken, as was also concluded in [1].

\section{Summary}

Very recently, the singular parts of four-particle amplitudes were bootstrapped in [1] by demanding that they factorise into a product of on-shell three-particle amplitudes on simple poles. In that work, consistent factorisation was implemented directly without making use of BCFW momentum shifts. In this short note, we have shown that the same results can be derived by using BCFW shifts to automate consistent factorisation. We presented full details for the illustrative cases of single spin- $S$ particle amplitudes but have also checked that the procedure produces the expected results for Compton scattering, and its gravitational analogue, as well as for scalars or photons coupled minimally to gravity. For single spin- $S$ particles, the boostless bootstrap teaches us that the leading three-particle couplings for a photon must vanish, the leading three-particle couplings for a graviton must be those of GR, while massless higher-spinning particles do not self-interact. For photon Compton scattering, boost-breaking interactions between the photon, scalars and spin- $1 / 2$ fermions are allowed and can be described by Lagrangians with generalised boostbreaking gauge redundancies. For gravitational Compton scattering, all couplings must reduce to their boost-invariant counter-parts with universal couplings of all particles to gravity. Finally, scalars and photons that are minimally coupled to gravity are forced to have Poincaré invariant self-interactions (constant or vanishing, respectively for the scalar and the photon). For full details see [1].

Although the theories we have considered are not a priori constructible, in the sense that the boundary terms do not necessarily vanish at large $z$, we have still been able to use BCFW shifts to constrain the three-particle couplings contributing to particle exchange.

\footnotetext{
${ }^{8}$ The spinor helicity parts of the $S=1$ three-particle amplitudes are odd under the exchange of identical particles so if the amplitudes are to be even by Bose symmetry, the $F$ 's must be alternating.

${ }^{9}$ For $S=2$, the spinor parts of the three-particle amplitudes are even under the exchange of identical particles and so the $F$ 's are symmetric polynomials.
} 
This does mean, of course, that the three-particle amplitudes themselves do not fully fix the four-particle ones. Indeed, all of the four-particle amplitudes we have constructed are defined up to the presence of "contact" terms that are regular for all kinematic configurations. It would be very interesting to investigate the possibility of using generalised momentum shifts, possibly along the lines of [41], to recursively derive exact higher-point amplitudes even if only for a subset of boost-breaking theories. It would also be very interesting to investigate the generalised on-shell recursion relations introduced in [42], where boundary terms are fixed with additional knowledge of a subset of the zeros of the deformed amplitude, in our boost-breaking setting.

\section{Acknowledgments}

We would like to thank Paolo Benincasa, Tanguy Grall, Sadra Jazayeri and Enrico Pajer for useful discussions and comments on a draft of this paper. D.S. has been supported in part by the research program VIDI with Project No. 680-47-535, which is (partly) financed by the Netherlands Organisation for Scientific Research (NWO). J.S. has been supported by a grant from STFC.

Open Access. This article is distributed under the terms of the Creative Commons Attribution License (CC-BY 4.0), which permits any use, distribution and reproduction in any medium, provided the original author(s) and source are credited.

\section{References}

[1] E. Pajer, D. Stefanyszyn and J. Supeł, The boostless bootstrap: amplitudes without Lorentz boosts, JHEP 12 (2020) 198 [arXiv:2007.00027] [INSPIRE].

[2] H. Elvang and Y.-T. Huang, Scattering amplitudes, arXiv:1308.1697 [INSPIRE].

[3] C. Cheung, TASI lectures on scattering amplitudes, in Proceedings, Theoretical Advanced Study Institute in elementary particle physics: anticipating the next discoveries in particle physics (TASI 2016), Boulder, CO, U.S.A., 6 June-1 July 2016, R. Essig and I. Low eds., World Scientific, Singapore (2018), pg. 571 [arXiv: 1708.03872] [INSPIRE].

[4] P. Benincasa, New structures in scattering amplitudes: a review, Int. J. Mod. Phys. A 29 (2014) 1430005 [arXiv: 1312.5583] [INSPIRE].

[5] S. Weinberg, Photons and gravitons in S-matrix theory: derivation of charge conservation and equality of gravitational and inertial mass, Phys. Rev. 135 (1964) B1049 [INSPIRE].

[6] S. Weinberg, Photons and gravitons in perturbation theory: derivation of Maxwell's and Einstein's equations, Phys. Rev. 138 (1965) B988 [INSPIRE].

[7] R. Britto, F. Cachazo, B. Feng and E. Witten, Direct proof of tree-level recursion relation in Yang-Mills theory, Phys. Rev. Lett. 94 (2005) 181602 [hep-th/0501052] [InSPIRE].

[8] P. Benincasa and F. Cachazo, Consistency conditions on the S-matrix of massless particles, arXiv:0705.4305 [INSPIRE].

[9] N. Arkani-Hamed, T.-C. Huang and Y.-T. Huang, Scattering amplitudes for all masses and spins, arXiv: 1709.04891 [INSPIRE]. 
[10] D.A. McGady and L. Rodina, Higher-spin massless S-matrices in four-dimensions, Phys. Rev. D 90 (2014) 084048 [arXiv:1311.2938] [INSPIRE].

[11] P.C. Schuster and N. Toro, Constructing the tree-level Yang-Mills S-matrix using complex factorization, JHEP 06 (2009) 079 [arXiv:0811.3207] [INSPIRE].

[12] N. Arkani-Hamed, D. Baumann, H. Lee and G.L. Pimentel, The cosmological bootstrap: inflationary correlators from symmetries and singularities, JHEP 04 (2020) 105 [arXiv: 1811.00024] [INSPIRE].

[13] D. Baumann, C. Duaso Pueyo, A. Joyce, H. Lee and G.L. Pimentel, The cosmological bootstrap: weight-shifting operators and scalar seeds, JHEP 12 (2020) 204 [arXiv: 1910.14051] [INSPIRE].

[14] N. Arkani-Hamed and J. Maldacena, Cosmological collider physics, arXiv:1503.08043 [INSPIRE].

[15] C. Sleight, A Mellin space approach to cosmological correlators, JHEP 01 (2020) 090 [arXiv: 1906.12302] [INSPIRE].

[16] C. Sleight and M. Taronna, Bootstrapping inflationary correlators in Mellin space, JHEP 02 (2020) 098 [arXiv: 1907.01143] [INSPIRE].

[17] D. Baumann, C. Duaso Pueyo, A. Joyce, H. Lee and G.L. Pimentel, The cosmological bootstrap: spinning correlators from symmetries and factorization, arXiv:2005.04234 [INSPIRE].

[18] D. Green and E. Pajer, On the symmetries of cosmological perturbations, JCAP 09 (2020) 032 [arXiv: 2004.09587] [INSPIRE].

[19] I. Mata, S. Raju and S. Trivedi, CMB from CFT, JHEP 07 (2013) 015 [arXiv:1211.5482] [INSPIRE].

[20] A. Ghosh, N. Kundu, S. Raju and S.P. Trivedi, Conformal invariance and the four point scalar correlator in slow-roll inflation, JHEP 07 (2014) 011 [arXiv:1401.1426] [INSPIRE].

[21] N. Kundu, A. Shukla and S.P. Trivedi, Constraints from conformal symmetry on the three point scalar correlator in inflation, JHEP 04 (2015) 061 [arXiv: 1410.2606] [INSPIRE].

[22] N. Arkani-Hamed, P. Benincasa and A. Postnikov, Cosmological polytopes and the wavefunction of the universe, arXiv:1709.02813 [INSPIRE].

[23] N. Arkani-Hamed and P. Benincasa, On the emergence of Lorentz invariance and unitarity from the scattering facet of cosmological polytopes, arXiv:1811.01125 [INSPIRE].

[24] P. Benincasa, From the flat-space S-matrix to the wavefunction of the universe, arXiv: 1811.02515 [INSPIRE].

[25] P. Benincasa, Cosmological polytopes and the wavefuncton of the universe for light states, arXiv: 1909. 02517 [INSPIRE].

[26] A. Hillman, Symbol recursion for the $d S$ wave function, arXiv:1912.09450 [INSPIRE].

[27] P. Benincasa, A.J. McLeod and C. Vergu, Steinmann relations and the wavefunction of the universe, Phys. Rev. D 102 (2020) 125004 [arXiv:2009.03047] [INSPIRE].

[28] H. Goodhew, S. Jazayeri and E. Pajer, The cosmological optical theorem, arXiv:2009.02898 [INSPIRE]. 
[29] J.M. Maldacena and G.L. Pimentel, On graviton non-Gaussianities during inflation, JHEP 09 (2011) 045 [arXiv: 1104.2846] [INSPIRE].

[30] S. Raju, New recursion relations and a flat space limit for AdS/CFT correlators, Phys. Rev. D 85 (2012) 126009 [arXiv:1201.6449] [INSPIRE].

[31] M.P. Hertzberg, J.A. Litterer and M. Sandora, Symmetries from locality. II. Gravitation and Lorentz boosts, Phys. Rev. D 102 (2020) 025023 [arXiv: 2005.01744] [InSPIRE].

[32] J. Khoury, G.E.J. Miller and A.J. Tolley, On the origin of gravitational Lorentz covariance, Class. Quant. Grav. 31 (2014) 135011 [arXiv: 1305.0822] [INSPIRE].

[33] M.P. Hertzberg and J.A. Litterer, Symmetries from locality. III. Massless spin-2 gravitons and time translations, Phys. Rev. D 102 (2020) 085007 [arXiv: 2008.06510] [INSPIRE].

[34] M.P. Hertzberg and M. Sandora, Special relativity from soft gravitons, Phys. Rev. D 96 (2017) 084048 [arXiv: 1704.05071] [INSPIRE].

[35] H.K. Dreiner, H.E. Haber and S.P. Martin, Two-component spinor techniques and Feynman rules for quantum field theory and supersymmetry, Phys. Rept. 494 (2010) 1 [arXiv:0812.1594] [INSPIRE].

[36] R. Britto, F. Cachazo and B. Feng, New recursion relations for tree amplitudes of gluons, Nucl. Phys. B $\mathbf{7 1 5}$ (2005) 499 [hep-th/0412308] [INSPIRE].

[37] P. Benincasa, C. Boucher-Veronneau and F. Cachazo, Taming tree amplitudes in general relativity, JHEP 11 (2007) 057 [hep-th/0702032] [INSPIRE].

[38] C. Cheung, K. Kampf, J. Novotny, C.-H. Shen and J. Trnka, On-shell recursion relations for effective field theories, Phys. Rev. Lett. 116 (2016) 041601 [arXiv: 1509.03309] [InSPIRE].

[39] A. Padilla, D. Stefanyszyn and T. Wilson, Probing scalar effective field theories with the soft limits of scattering amplitudes, JHEP 04 (2017) 015 [arXiv: 1612.04283] [INSPIRE].

[40] B. Feng, K. Zhou, C. Qiao and J. Rao, Determination of boundary contributions in recursion relation, JHEP 03 (2015) 023 [arXiv: 1411.0452] [INSPIRE].

[41] S. Raju, Four point functions of the stress tensor and conserved currents in $A d S_{4} / C F T_{3}$, Phys. Rev. D 85 (2012) 126008 [arXiv:1201.6452] [INSPIRE].

[42] P. Benincasa and E. Conde, On the tree-level structure of scattering amplitudes of massless particles, JHEP 11 (2011) 074 [arXiv:1106.0166] [INSPIRE]. 